\title{
Estudio comparativo entre los biómetros ópticos IOL Master 500 versus IOL Master 700 en pacientes con catarata y análisis de repetibilidad
}

\section{Comparative analysis and repeatability assessment of IOL Master $\mathbf{5 0 0}$ versus IOL Master $\mathbf{7 0 0}$ biometry in cataract patients}

\author{
Roxana Saucedo-Urdapilleta', Sara González-Godínez', Mariana Mayorquín-Ruiz¹, \\ Eduardo Moragrega-Adame ${ }^{1}$, Cecilio Velasco-Barona ${ }^{2}$ y Roberto González-Salinas ${ }^{3 *}$ \\ ${ }^{1}$ Departamento de Ecografía; ${ }^{2}$ Departamento de Segmento Anterior; ${ }^{3}$ Departamento de Investigación. Asociación para Evitar la Ceguera en México \\ IAP, Ciudad de México, México
}

\section{Resumen}

Objetivo: Evaluar la repetibilidad del IOL Master 700, biómetro basado en tomografía de coherencia óptica de fuente de barrido, así como del IOL Master 500, biómetro basado en interferometría de coherencia parcial, además de evaluar su correlación y concordancia, en pacientes candidatos a cirugía de catarata. Diseño del estudio: Comparativo y prospectivo. Métodos: Se obtuvieron los valores de la longitud axial $(L A)$, queratometrías (K), profundidad de cámara anterior (ACD) y distancia blanco-blanco (WTW) con el IOL Master 500, y se compararon con los obtenidos con el IOL Master 700. Se realizó la prueba de $t$ de Student para comparar variables continuas. La correlación de Pearson y el análisis de Bland-Altman se usaron para describir la correlación y el límite de acuerdo entre plataformas. Resultados: El estudio incluyó 55 ojos de 55 pacientes con edad promedio de $69 \pm 11$ años de edad. La diferencia promedio de $L A, K, A C D$ y WTW fue de $0.16 \mathrm{~mm} \pm$ 2.30, $0.06 \mathrm{D} \pm 0.38,0.02 \mathrm{~mm} \pm 0.12$ y $0.15 \mathrm{~mm} \pm 0.67$, respectivamente. Existen diferencias estadísticamente significativas entre los biómetros para dos parámetros: $L A(p=0.0003)$ y $A C D(p=0.038)$. Conclusiones: La biometría óptica mostró una alta repetibilidad para todos los parámetros en ambos equipos. Los valores de $K$ y WTW mostraron un buen nivel de acuerdo; sin embargo, la LA y la ACD mostraron diferencias estadísticamente significativas entre biómetros ópticos.

Palabras clave: Tomografía de coherencia óptica de fuente de barrido. IOL Master 700. Tomografía óptica de coherencia parcial. IOL Master 500. Biometría.

\section{Abstract}

Purpose: To assess the repeatability of the swept-source optical coherence tomography (SS-OCT) biometer (IOL Master 700) and evaluate its agreement with a partial coherence interferometry (PCI) biometer (IOL Master 500) in patients undergoing routine cataract surgery. Design: This is a prospective, comparative study. Methods: Axial length (AL), keratometry ( $k$ ), anterior chamber depth (ACD) and white-to-white distance (WTW) values were obtained by PCl-based biometer and compared with those obtained by SS-OCT. A Student's t-test was used for comparison of continuous variables. Pearson's

\section{Correspondencia:}

*Roberto González-Salinas

Vicente García Torres, 46

Barrio San Lucas, Del. Coyoacán Fecha de recepción: 09-07-2018

C.P. 04030, Ciudad de México, México

E-mail: dr.gonzalezsalinas@apec.com.mx

0187-4519/○ 2019 Sociedad Mexicana de Oftalmología. Publicado por Permanyer México. Este es un artículo Open Access bajo la licencia CC BY-NC-ND (http://creativecommons.org/licenses/by-nc-nd/4.0/).
Fecha de recepción: 09-07-2018

DOI: 10.24875/RMO M19000067
Disponible en internet: 01-05-2019 Rev Mex Oftalmol. 2019;93(3):130-136

www.rmo.com.mx 
correlation coefficient and Bland-Altman plots were obtained to describe the correlation and limits of agreement between platforms. Results: The study included 55 eyes with a mean age of $69 \pm 11$ years of age. The mean differences between swept-source and PCl-based biometry for AL, keratometry, ACD and WTW distance were $0.16 \mathrm{~mm} \pm 2.30,0.06 \mathrm{D} \pm 0.38$, $0.02 \mathrm{~mm} \pm 0.12$ and $0.15 \mathrm{~mm} \pm 0.67$, respectively. There were statistically significant differences between biometers in two parameters: $A L(p=0.0003)$ and ACD $(p=0.038)$. Conclusion: SS-OCT biometry showed high repeatability for all biometric parameters. Keratometry and WTW distance values showed a good level of agreement between SS-OCT and PCI-based biometry. However, AL and ACD measurements showed statistically significant differences between the two optical biometers.

Key words: Swept-source optical coherence tomography. IOL Master 700. Partial coherence interferometry. IOL Master 500. Biometry.

\section{Introducción}

La biometría por ultrasonido requiere de contacto directo con estructuras oculares para medir correctamente la longitud axial ${ }^{1}$. La biometría óptica, basada en tecnología modo $A$, fue introducida con el equipo IOL Master ${ }^{2}$. La interferometría de coherencia parcial $(\mathrm{PCl})$ no requiere contacto con el ojo, lo que evita posibles riesgos de patología corneal y, además, obtiene mejores resultados en la toma de longitud axial en comparación con la biometría por ultrasonido ${ }^{2,3}$.

La biometría óptica ha sido descrita como un método útil para el cálculo de la lente intraocular (LIO) en el $90 \%$ a $95 \%$ de los casos de catarata (exceptuando casos de cataratas densas, pacientes con mala fijación, condensaciones o hemorragia vítrea, opacidades de la cápsula posterior, entre otras) ${ }^{2}$. El IOL Master 500 mide la longitud axial (LA), usando $\mathrm{PCl}$, y la de las queratometrías (K), la distancia blanco-blanco (WTW) y la profundidad de cámara anterior (ACD) desde el epitelio corneal a la superficie anterior del cristalino, usando análisis de imagen. Cada medida requiere que el instrumento esté alineado con el eje visual ${ }^{3-5}$.

La técnica de medición de la mayoría de los equipos disponibles en el mercado actualmente está basada en interferometría de dominio de tiempo ${ }^{3}$. Actualmente, existe una nueva tomografía de coherencia óptica (OCT), llamada de fuente de barrido (OCT-SS). Esta tecnología usa el IOL Master $700^{6}$, equipo que mide la $\mathrm{LA}$, valores de las $\mathrm{K}, \mathrm{ACD}$, WTW, grosor del cristalino y grosor corneal central ${ }^{2,3}$.

El propósito de este estudio es el de analizar la repetibilidad de la biometría con OCT-SS y, adicionalmente, estimar el nivel de acuerdo para las mediciones biométricas (LA, ACD, WTW y K) usando la OCT-SS, así como la OCT con dominio de tiempo en pacientes sometidos a cirugía de catarata de rutina.

\section{Métodos}

El Comité Interno de Evaluación de Trabajos de la Asociación para Evitar la Ceguera en México aprobó este estudio. Todos los procedimientos se realizaron conforme a la Declaración de Helsinki. Se obtuvo un consentimiento informado por escrito firmado por todos los pacientes que participaron en el estudio después de explicarles los procedimientos y posibles complicaciones.

Es un estudio prospectivo y comparativo en el que se incluyeron pacientes que acudieron al Hospital Dr. Luis Sánchez Bulnes de la a Asociación para Evitar la Ceguera en México, de cualquier sexo, mayores de 18 años, con diagnóstico de cualquier tipo de catarata, de cualquier grado según la escala fotográfica lens opacities classification system (LOCS) III $^{7}$, que evalúa esclerosis nuclear (clasificación 0 a 6), color de opacidad nuclear (clasificación 0 a 6), opacidad cortical (clasificación 0 a 5) y opacidad subcapsular posterior (clasificación 0 a 5), en los que sea posible realizar biomicroscopia en lámpara de hendidura.

Los criterios de exclusión incluyeron cualquier patología corneal, usuarios de lentes de contacto, glaucoma, traumatismo ocular o cirugía, así como el uso de cualquier tratamiento ocular diferente a lubricante durante un periodo mínimo de un mes.

\section{Análisis estadístico}

Se realizó un análisis descriptivo de las variables incluidas en los datos demográficos (incluyendo edad y género). Para las comparaciones de medias entre variables continuas con distribución normal, se realizó una Prueba $t$ de Student pareada, y para variables continuas no normales, se usó la prueba de los rangos con signo de Wilcoxon. Se obtuvo el coeficiente de correlación de Pearson (r) entre grupos para todas las variables. Adicionalmente, se evaluó la concordancia 

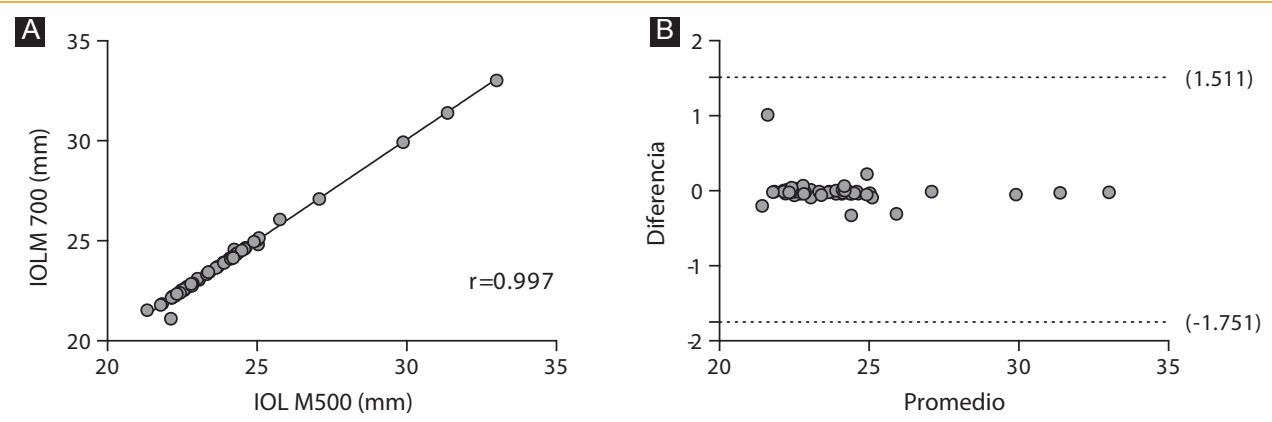

Figura 1. A: Gráfica de la correlación de Pearson para los valores de la longitud axial entre plataformas $(r=0.997$; $\mathrm{p}<0.0001)$. B: Gráfica de Bland-Altman entre la diferencia vs. promedio de los valores de la longitud axial con un nivel de acuerdo del $95 \%$.

o nivel de acuerdo entre las plataformas a través del análisis de Bland-Altman ${ }^{8}$.

La distribución de las bases de datos se revisó usando el test de Kolmogorov-Smirnov. Para determinar la repetibilidad de la OCT-SS, se usaron dos indicadores: la desviación estándar y el coeficiente de variación, con un modelo mixto para 3 medidas repetidas de 2 vías.

Se estimó necesario un tamaño de muestra de 55 pacientes para comparar las medias entre dos grupos, con nivel de significancia de 0.05 ( $\square$ de dos colas, $\beta$, de 0.2 ), poder del $80 \%$ y 0.95 de desviación estándar en la población. Se llevó a cabo el análisis estadístico usando los programas Statistical Package for Social Sciences, versión 20.0 (SPSS, Inc., Chicago, IL) y el Graph-Pad Prism 2015 versión 6.0.

\section{Resultados}

En este estudio se evaluaron 55 ojos de 55 pacientes, $61.8 \%(n=34)$ del sexo femenino y $32.8 \%(n=21)$ del sexo masculino, con una edad promedio de $69 \pm$ 11.12 años (rango 58 a 80 años); 26 (47\%) ojos derechos y 29 (53\%) ojos izquierdos.

Hubo 16 ojos con opacidad nuclear (NO) grado 1, 20 ojos NO2, 8 ojos NO3, 8 ojos NO4, y ningún ojo NO5 o NO6; 23 ojos tenían opacidad posterior ( $\mathrm{P} 1=9$ ojos, $\mathrm{P} 2=4$ ojos, $\mathrm{P} 3=8$ ojos, $\mathrm{P} 4=1$ ojo, $\mathrm{P} 5=1$ ojo) y 23 ojos con cataratas corticales (C1 $=6$ ojos, $\mathrm{C} 2=12$ ojos, C3 = 1 ojo, C4 = 3 ojos, C5 = 1 ojo).

La desviación estándar entre sujetos y el coeficiente de variación de los parámetros obtenidos con el IOL Master 500 y el IOL Master 700 se muestran en la tabla 1.

Los valores de LA, K, ACD y WTW obtenidos por ambos biómetros se presentan en la tabla 2 .
Tabla 1. Desviación estándar de repetibilidad y coeficiente de variación por parámetros entre biómetros

\begin{tabular}{|l|c|c|c|c|}
\hline Parámetro & \multicolumn{2}{|c|}{$\begin{array}{c}\text { Desviación } \\
\text { estándar }\end{array}$} & \multicolumn{2}{c|}{$\begin{array}{c}\text { Coeficiente de } \\
\text { variación (\%) }\end{array}$} \\
\cline { 2 - 5 } & PCI & OCT-SS & \multicolumn{1}{c|}{ PCI } & OCT-SS \\
\hline AL (mm) & 2.18 & 2.44 & 0.09 & 0.10 \\
\hline Km (D) & 1.65 & 1.60 & 0.03 & 0.03 \\
ACD (mm) & 0.43 & 0.43 & 0.14 & 0.13 \\
WTW (mm) & 0.47 & 0.52 & 0.03 & 0.04 \\
GCC (m) & - & 36.21 & - & 0.06 \\
GC (mm) & - & 0.45 & - & 0.10 \\
\hline
\end{tabular}

ACD: profundidad de cámara anterior; AL: longitud axial; GC: grosor de cristalino; GCC: grosor corneal central; K: queratometría promedio; WTW: distancia blanco-blanco; $\mathrm{PCl}$ = interferometría de coherencia parcial (IOLMaster 500); OCT: tomografía de secuencia óptica de fuente de (IOLMaster 700).

El coeficiente de correlación de Pearson (r), así como la gráfica del análisis de Bland-Altman, donde se muestra la diferencia versus el promedio de las medidas de longitud axial, obtenidas con ambos biómetros, se describen en la figura 1. Adicionalmente, se observó una diferencia estadísticamente significativa entre los valores obtenidos con el biómetro basado en OCT-SS, que fueron más largos en comparación con los obtenidos con el biómetro basado en interferometría de coherencia parcial $(p<0.0003)$.

En la figura 2 se muestran las gráficas de la correlación de Pearson ( $r$ ) entre los valores de las K obtenidos con ambos biómetros, así como el análisis de Bland-Altman. No existe diferencia estadísticamente significativa entre los valores obtenidos entre grupos $(p=0.271)$. 


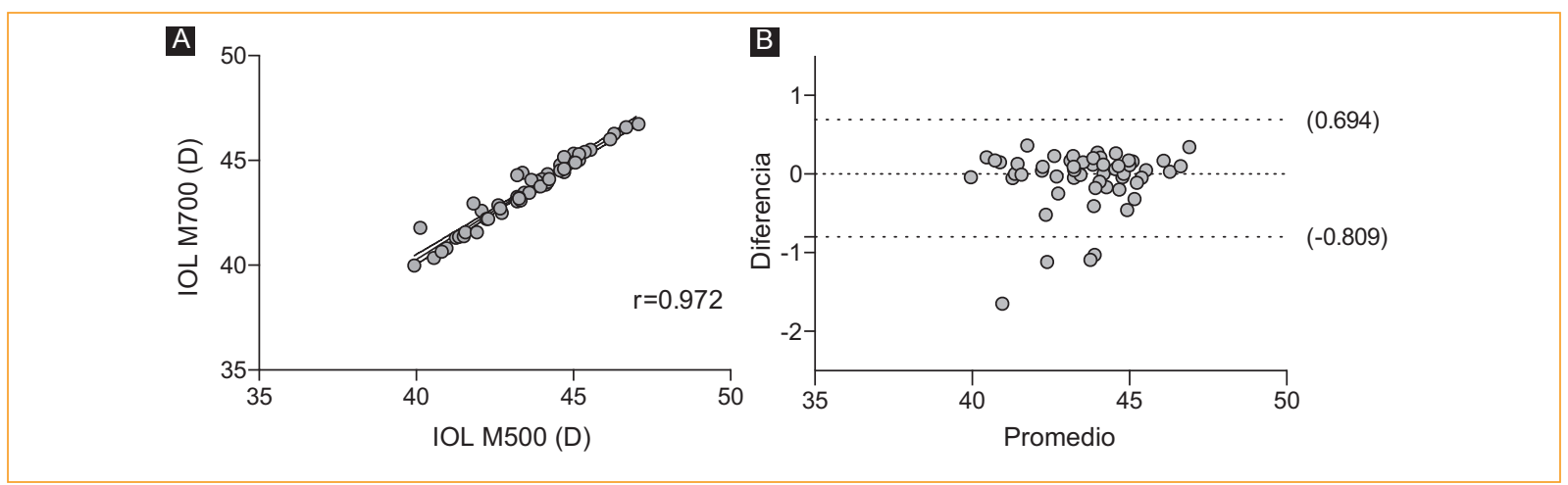

Figura 2. A: Gráfica de la correlación de Pearson para los valores de las queratometrías entre plataformas $(r=0.972$; $\mathrm{p}<0.0001)$. B: Gráfica de Bland-Altman entre la diferencia vs. promedio de los valores de las queratometrías con un nivel de acuerdo del $95 \%$.

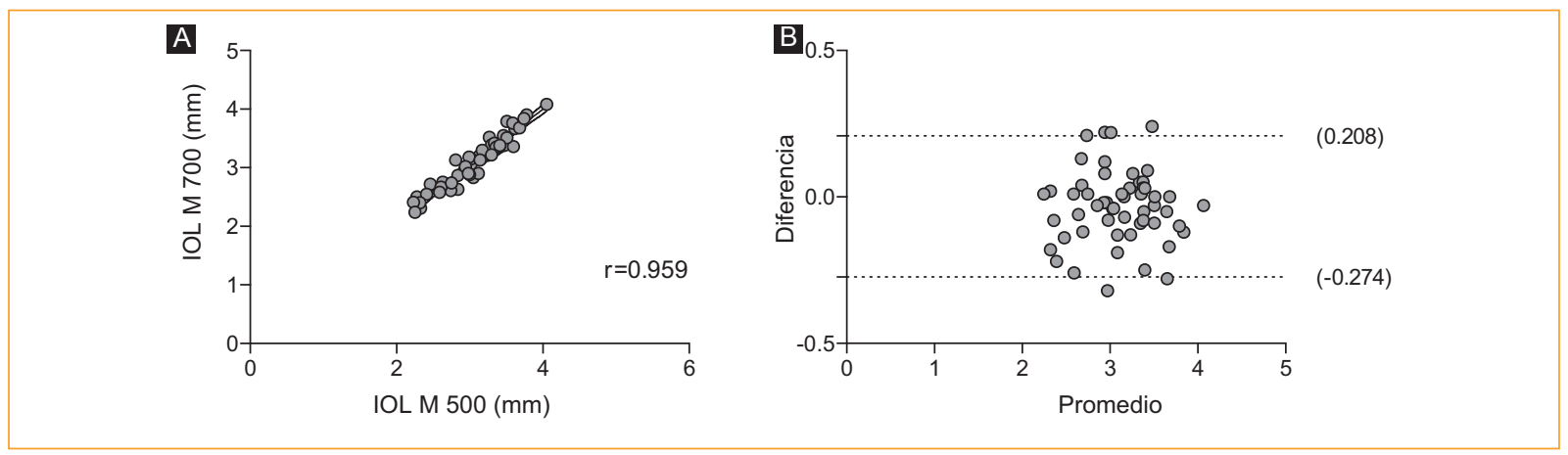

Figura 3. A: Gráfica de la correlación de Pearson para los valores de la profundidad de cámara anterior entre plataformas $(r=0.959 ; p<0.0001)$. B: Gráfica de Bland-Altman entre la diferencia vs. promedio de los valores de la profundidad de cámara anterior con un nivel de acuerdo del $95 \%$.

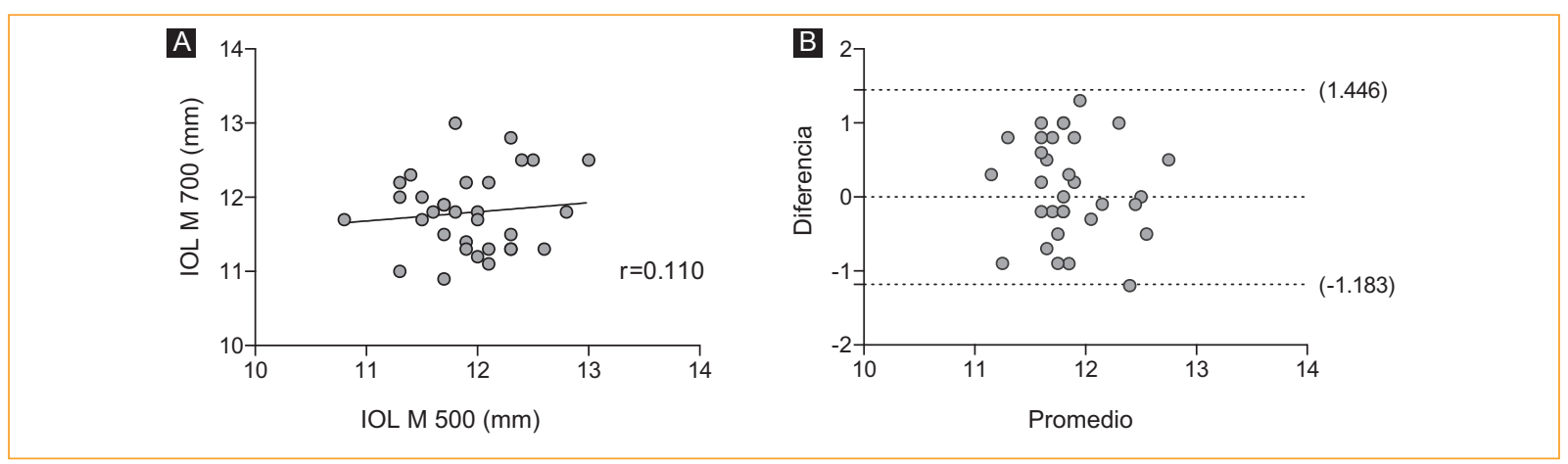

Figura 4. A: Gráfica de la correlación de Pearson para los valores de la distancia blanco-blanco entre plataformas $(r=0.110 ; p=0.545)$. B: Gráfica de Bland-Altman entre la diferencia vs. promedio de los valores de la de la distancia blanco-blanco con un nivel de acuerdo del $95 \%$.

La figura 3 muestra las gráficas de correlación entre las medidas de ACD obtenidas con IOL Master 500 e IOL Master 700, así como el análisis de Bland-Altman.
Las medidas de la ACD adquiridas con el IOL Master 700 fueron significativamente más largas que las obtenidas con IOL Master $500(p<0.038)$. 
Tabla 2. Medidas obtenidas por el IOL Master 500 y el IOLMaster 700

\begin{tabular}{|l|c|c|c|c|c|}
\hline \multirow{2}{*}{ Parámetro } & \multicolumn{2}{|c|}{ IOL Master 500} & \multicolumn{2}{c|}{ IOL Master 700} \\
\cline { 2 - 6 } & Promedio \pm DE & Rango & Promedio \pm DE & \multicolumn{1}{c|}{ Rango } \\
\hline AL (mm) & $23.94 \pm 2.16$ & $21.33,33.00$ & $24.10 \pm 2.44$ & $21.11,32.02$ & $0.0003^{*}$ \\
\hline K (D) & $43.55 \pm 1.65$ & $39.94,47.07$ & $43.61 \pm 1.60$ & $39.98,46.73$ & 0.271 \\
\hline ACD (mm) & $3.08 \pm 0.43$ & $2.23,4.05$ & $3.11 \pm 0.43$ & $2.24,4.08$ & $0.038^{*}$ \\
\hline WTW (mm) & $11.93 \pm 0.47$ & $10.08,13.00$ & $11.78 \pm 0.52$ & $10.09,13.00$ & 0.276 \\
\hline
\end{tabular}

*Estadísticamente significativo.

ACD: profundidad de cámara anterior; AL: longitud axial; DE: desviación estándar; K: queratometría; WTW: distancia blanco-blanco.

Tabla 3. Resultados comparativos entre autores

\begin{tabular}{|c|c|c|c|c|}
\hline & AL $(\mathrm{mm})$ & K (D) & ACD (mm) & WTW $(\mathrm{mm})$ \\
\hline Shammas, et al. ${ }^{2}$ & 0.02 & 0.18 & 0.06 & $-\cdots--$ \\
\hline Srivannaboon, et al. ${ }^{12}$ & 0.02 & 0.01 & 0.04 & 0.10 \\
\hline Akman, et al. ${ }^{9}$ & 0.005 & 0.05 plana 0.08 curva & 0.08 & ----- \\
\hline Kurian, et al. ${ }^{11}$ & 0.01 & 0.02 & 0.03 & ----- \\
\hline Saucedo-Urdapilleta, et al. 2019 & 0.16 & 0.06 & 0.02 & 0.15 \\
\hline
\end{tabular}

ACD: profundidad de cámara anterior; AL: Iongitud axial; K: queratometría promedio; WTW: distancia blanco-blanco.

La figura 4 describe la gráfica de correlación entre las WTW obtenidas entre grupos, así como el análisis de Bland-Altman. No se encontraron diferencias estadísticamente significativas entre los valores de la WTW entre grupos $(p=0.276)$.

\section{Discusión}

El advenimiento de nuevas tecnologías en el diseño de lentes intraoculares y técnicas quirúrgicas ha aumentado la expectativa de los pacientes para obtener una visión postoperatoria sin necesidad de una corrección refractiva, por tanto, el cálculo adecuado del poder de la LIO tiene un papel fundamental ${ }^{9}$.

El IOL Master, basado en interferometría de coherencia parcial, fue el primer biómetro disponible. Actualmente se considera el estándar de oro para la valoración preoperatoria en cirugía de catarata ${ }^{10}$. En nuestro estudio, el biómetro basado en OCT-SS demostró excelente repetibilidad para todos los parámetros y un buen nivel de acuerdo para los valores de las $\mathrm{K}$ y la WTW, en concordancia con lo reportado previamente por Kurian, et al. ${ }^{11}$ Además, las medidas de las $\mathrm{K}$ y la WTW obtenidas en este estudio demostraron una buena correlación entre equipos, en concordancia con lo reportado recientemente por Akman, et al. ${ }^{9}$ y Srivannaboon, et al. ${ }^{12}$; sin embargo, hubo una diferencia estadísticamente significativa con respecto a la LA y la ACD.

Los valores de LA obtenidos con IOL Master 500 evidenciaron diferencias estadísticamente significativas en comparación con los obtenidos con el IOL Master $700(p=0.0003)$. La diferencia promedio encontrada en nuestro estudio coincide con lo reportado por Shammas, et al. ${ }^{2}$. Basándose en cálculos ópticos, una diferencia de $0.16 \mathrm{~mm}$ resultaría en un error en la refracción final de $0.40 \mathrm{D}$ (en ojos con LA y $\mathrm{K}$ promedio), lo que podría ser clínicamente significativo y, a diferencia de lo mencionado por otros autores, debemos considerarlo. Nuestros resultados son contrastados con estudios previos en la tabla 3.

Las diferencias con respecto a la LA se deben probablemente al mecanismo por el que la OCT-SS mide esta variable; la OCT-SS tiene una profundidad de escaneo de $44 \square \mathrm{m}$, con $22 \square \mathrm{m}$ de resolución en los tejidos, lo que permite una rápida adquisición de cortes (2000 A-scans/s), a diferencia del IOL Master 500 que utiliza un láser diodo de $780 \mathrm{~nm}^{5}$. 
No existe diferencia significativa entre los valores de las $\mathrm{K}$ medidas entre ambos equipos $\mathrm{y}$, como se mencionó previamente, las medidas tomadas por el IOL Master 700 demostraron una alta correlación con las tomadas con el IOL Master 500. La diferencia entre promedios fue de $0.06 \mathrm{D}$, lo que resulta en un error refractivo postquirúrgico de aproximadamente $0.04 \mathrm{D}$, valor sin significancia clínica.

Con respecto a la profundidad de cámara anterior, existe una diferencia estadísticamente significativa $(p=0.038$ ) entre medidas biométricas, aunque la diferencia entre promedios fue solo $0.02 \mathrm{~mm}$. Esto probablemente se debe a que el IOL Master 500 basa su medición en un corte de hendidura a través de la cámara anterior, lo que pudiera significar en una medición no centrada ${ }^{13,14}$. En comparación, el IOL Master 700 mide la ACD, el grosor de cristalino y el grosor corneal central en una sola imagen de OCT, alineada con el eje visual. Este prámetro no afecta el cálculo de la LIO cuando se usan la mayoría de las fórmulas ${ }^{13-15}$; sin embargo, cuando usamos la fórmula de cuarta generación Haigis ${ }^{16}$, una diferencia de 0.06 mm en la ACD solo cambia la refracción final en $0.05 \mathrm{D}^{17}$, lo que no es clínicamente significativo; sin embargo, se puede afirmar que el estudio del grosor corneal y del cristalino es importante para las nuevas fórmulas y útil en el futuro del cálculo del poder de la LIO.

La distancia blanco-blanco, solo es necesaria en algunas fórmulas y es particularmente útil en el cálculo de lentes fáquicos ${ }^{18,19}$.

Aunque la repetibilidad del grosor corneal y del cristalino con el IOL Master 700 es alta y fácil de medir, no se considera en las fórmulas usadas comúnmente; sin embargo, es relevante para fórmulas recientemente desarrolladas, por ejemplo Holladay 2 y Olsen ${ }^{20,21}$.

Una limitación que tiene el estudio es el tamaño limitado de pacientes, por lo que una muestra más grande sería conveniente para confirmar la precisión de las medidas. Además, no se incluyeron pacientes con hipermetropía u ojos cortos ni pacientes con patología ocular diferente de las cataratas.

\section{Conclusiones}

El biómetro óptico basado en OCT-SS ha probado ser una herramienta de gran valor para realizar el cálculo de poder de la LIO previo a cirugía de catarata en nuestra población. Adicionalmente, el nivel de acuerdo entre biómetros fue bueno demostrando una alta repetibilidad.

\section{Conflicto de intereses}

Los autores declaran no tener ningún conflicto de intereses.

\section{Responsabilidades éticas}

Protección de personas y animales. Los autores declaran que para esta investigación no se han realizado experimentos en seres humanos ni en animales.

Confidencialidad de los datos. Los autores declaran que han seguido los protocolos de su centro de trabajo sobre la publicación de datos de pacientes.

Derecho a la privacidad y consentimiento informado. Los autores han obtenido el consentimiento informado de los pacientes y/o sujetos referidos en el artículo. Este documento obra en poder del autor de correspondencia.

\section{Bibliografía}

1. Grulkowski I, Liu JJ, Zhang JY, Potsaid B, Jayaraman V, Cable AE, Duker JS, Fujimoto JG. Reproducibility of a long-range swept-source optical coherence tomography ocular biometry system and comparison with clinical biometers. Ophthalmology. 2013;120(11):2184-90.

2. Shammas HJ, Hoffer KJ. Repeatability and reproducibility of biometry and keratometry measurements using a noncontact optical lowcoherence reflectometer and keratometer. Am J Ophthalmol. 2012; 153(1):55-61.

3. Lee AC, Qazi MA, Pepose JS. Biometry and intraocular lens power calculation. Curr Opin Ophthalmol. 2008;19(1):13-7.

4. Chen YA, Hirnschall N, Findl O. Evaluation of 2 new optical biometry devices and comparison with the current gold standard biometer. $\mathrm{J} \mathrm{Ca}$ taract Refract Surg. 2011;37(3):513-7.

5. Santodomingo-Rubido J, Mallen EA, Gilmartin B, Wolffsohn JS. A new non-contact optical device for ocular biometry. $\mathrm{Br} \mathrm{J}$ Ophthalmol. 2002; 86(4):458-62.

6. Kunert KS, Peter M, Blum M, Haigis W, Sekundo W, Schütze J, Büehren T. Repeatability and agreement in optical biometry of a new swept-source optical coherence tomography-based biometer versus partial coherence interferometry and optical low-coherence reflectometry. J Cataract Refract Surg. 2016;42(1):76-83

7. Karbassi M, Khu PM, Singer DM, Chylack JL. Evaluation of lens opacities classification system III applied at the slitlamp. Optom Vis Sci. 1993; 70(11):923-8.

8. Bland JM, Altman DG. Agreement between methods of measurement with multiple observations per individual. J Biopharm Stat. 2007;17(4): 571-82.

9. Akman A Asena L, Güngör SG. Evaluation and comparison of the new swept source OCT-based IOLMaster 700 with the IOLMaster $500 . \mathrm{Br} J$ Ophthalmol. 2016;100(9):1201-5.

10. Kaswin G, Rousseau A, Mgarrech M, Barreau E, Labetoulle M. Biometry and intraocular lens power calculation results with a new optical biometry device: comparison with the gold standard. J Cataract Refract Surg. 2014;40(4):593-600.

11. Kurian M, Negalur N, Das S, Puttaiah NK, Haria D, Thakkar MM. Biometry with a new swept-source optical coherence tomography biometer: Repeatability and agreement with an optical low-coherence reflectometry device. J Cataract Refract Surg. 2016;42(4):577-81.

12. Srivannaboon $S$, Chirapapaisan $C$, Chonpimai $P$, Loket $S$. Clinical comparison of a new swept-source optical coherence tomography-based optical biometer and a time-domain optical coherence tomography-based optical biometer. J Cataract Refract Surg. 2015;41(10):2224-32.

13. Holladay JT, Musgrove KH, Prager TC, Lewis JW, Chandler TY, Ruiz RS. A three-part system for refining intraocular lens power calculations. J Cataract Refract Surg. 1988:14(1):17-24.

14. Retzlaff JA, Sanders DR, Kraff MC. Development of the SRK/T intraocular lens implant power calculation formula. J Cataract Refract Surg. 1990;16(3):333-40. 
Rev Mex Oftalmol. 2019;93

15. Hoffer KJ. The Hoffer $Q$ formula: a comparison of theoretic and regression formulas. J Cataract Refract Surg. 1993;19(6):700-12.

16. Haigis W. Intraocular lens calculation in extreme myopia. J Cataract Refract Surg. 2009;35(5):906-11.

17. Vogel A, Dick HB, Krummenauer F. Reproducibility of optical biometry using partial coherence interferometry: intraobserver and interobserver reliability. J Cataract Refract Surg. 2001;27(12):1961-8.

18. Alfonso JF, Fernández-Vega L, Lisa $C$, Fernandes $\mathrm{P}$, Jorge J, Micó RM Central vault after phakic intraocular lens implantation: correlation with anterior chamber depth, white-to-white distance, spherical equivalent, and patient age. J Cataract Refract Surg. 2012;38(1):46-53.

19. Çağlar Ç, Kocamış Sİ, Demir E, Durmuş M. Comparison of the measurements of a novel optical biometry: Nidek AL-Scan with Sirius and a ultrasound biometry. International Ophthalmol. 2017;37(3):491-8.

20. Olsen T. Calculation of intraocular lens power: a review. Acta Ophthalmo Scand. 2007;85(5):472-85

21. Epitropoulos A. Axial length measurement acquisition rates of two optical biometers in cataractous eyes. Clin Ophthalmol. 2014;8:1369. 